\title{
Sepsis, meningitis and cerebral abscesses caused by Citrobacter koseri
}

\author{
Clara Vaz Marecos, ${ }^{1}$ Marta Ferreira, ${ }^{2}$ Maria Manuela Ferreira, ${ }^{2}$ Maria Rosalina Barroso ${ }^{2}$
}

1Paediatric Department, Professor Doutor Fernando Fonseca Hospital, EPE, Lisbon, Portugal;

${ }^{2}$ Neonatal Intensive Care Unit, Professor Doutor Fernando Fonseca Hospital, Amadora, Portugal

Correspondence toDr Clara Vaz Marecos, claravazmarecos@gmail.com

\begin{abstract}
Summary
After a 36-week diamniotic dichorionic gestation, an infant was delivered by elective caesarean section due to growth restriction and altered diastolic flow in the umbilical artery. Birth weight was $2140 \mathrm{~g}$. The patient was admitted for exclusive parenteral nutrition, with umbilical venous catheter placement. Sinus tachycardia and temperature instability with positive inflammatory markers occurred at $51 \mathrm{~h}$. Penicillin and gentamicin were started, but $6 \mathrm{~h}$ later septic shock with disseminated intravascular coagulation was noted. Vancomycin and meropenem were started and penicillin suspended. Citrobacter koseri was isolated from blood culture. Generalised clonic convulsions occurred on day 4 , and an electroencephalogram revealed severe encephalic dysfunction. Cerebrospinal fluid cytochemical analysis was suggestive of meningitis, although culture was negative. Cefotaxime was added to the drug regimen. Cranioencephalic MR showed a temporal abscess and diffuse hemispheric destruction, with no indications for neurosurgery. After 6 weeks of therapy, neuroimaging follow-up showed multiloculated cystic encephalomalacia. Currently, the patient is 14 months old with axial hypotonia and decreased movements. The source of infection has not been determined. Nosocomial infection cannot be excluded and vertical transmission is unlikely.
\end{abstract}

\section{BACKGROUND}

Newborns have the highest incidence of bacterial meningitis, with this infection being more common in the first month of life than at any other time. The global incidence is 0.25 , with one case per 1000 live births and 0.15 cases per 1000 full term births. ${ }^{1}$

Around $30 \%$ of newborns with sepsis have associated meningitis. The most common pathogens are group B Streptococcus, enteric bacilli and Listeria monocytogenes. Neonatal meningitis due to Citrobacter koseri is very rare. Although the source of neonatal infection cannot be identified in the majority of cases, vertical transmission during delivery may occur as well as nosocomial transmission from healthcare workers.

Citrobacter species are Gram-negative enteric bacilli found in the intestinal tract of humans and animals, and are also present in soil and water. The genus consists of three different species, one of which, $C$ koseri, is a particularly devastating, although rare, cause of meningitis. ${ }^{2}$ Meningitis due to this agent leads to intracerebral abscesses in most cases $(75 \%)$, with other complications including cerebral oedema, diffuse necrotising meningoencephalitis, ventriculitis, cerebritis, empyema, pneumatocephalus, cerebral infarction hydrocephalus and severe neurological impairment (50\% of cases). ${ }^{3}$ Seizures occur in $75 \%$ of cases. $^{3}$

The death rate is high (30\%), with the surviving infants having severe neurological sequelae, the most frequent $(80 \%)$ being mental retardation. ${ }^{4}$

\section{CASE PRESENTATION}

We report the case of a female newborn, the first twin of non-consanguineous healthy parents, who was born after a diamniotic dichorionic 36-week gestation with adequate prenatal care. Maternal vaginal screening for group B Streptococcus was positive at 35 weeks.

Prenatal ultrasound revealed asymmetric fetal growth since the 26th week of gestation, showing growth restriction of the first twin (10th percentile) with the second twin at the 50th percentile. The twins were delivered by elective caesarean section due to growth restriction and altered diastolic flow in the umbilical artery.

The Apgar score of the smaller infant was 9/10, birth weight was $2140 \mathrm{~g}$ (3rd-10th percentile), length was 41 $\mathrm{cm}(<3 \mathrm{rd}$ percentile) and head circumference $31.5 \mathrm{~cm}$ (10th percentile).

The newborn was admitted to the neonatal intensive care unit for exclusive parenteral feeding (nothing by mouth for $48 \mathrm{~h}$ ) due to diastolic flux alterations. On admission, the patient had a normal physical exam and laboratory evaluation. An umbilical venous catheter was placed.

On day 3 (51 h of age), the patient experienced tachycardia $(220 \mathrm{ppm})$ and temperature instability $\left(37.5^{\circ} \mathrm{C}\right)$ with a rise in inflammatory markers ( $\mathrm{C}$ reactive protein 1.89 $\mathrm{mg} / \mathrm{dl}$ ). An electrocardiogram showed sinus tachycardia. Penicillin and gentamicin were started.

Septic shock with disseminated intravascular coagulation occurred $6 \mathrm{~h}$ later and required ventilatory, inotropic and transfusional support. Vancomycin and meropenem were started and penicillin suspended.

On day 4, generalised tonic convulsions occurred. Electroencephalogram (EEG) showed severe encephalic dysfunction with global flattened electrogenesis and multifocal ictal and interictal paroxysmal activity at temporal regions bilaterally. Phenobarbital was started. A transfontanellar ultrasound (TFU) revealed no alterations on this date.

On day 6, $C$ koseri was isolated from the blood culture taken on day 3. $C$ koseri was sensitive to amoxicillin plus 


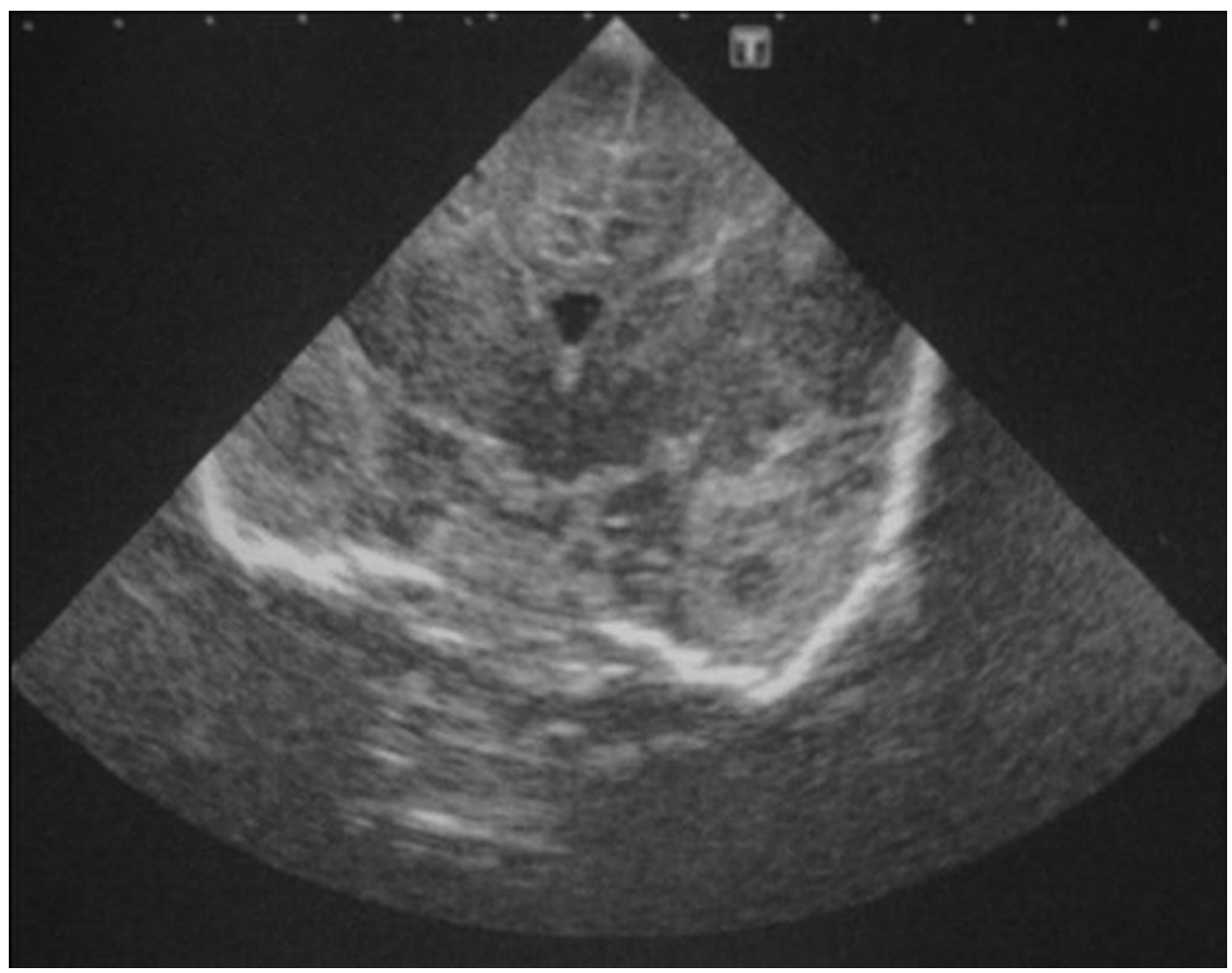

Figure 1 Transfontanellar ultrasound image showing temporal abscess.

clavulanic acid, cefradine, cefotaxime, cefuroxime, gentamicin, tobramycin, ciprofloxacin, cotrimoxazole, tazobac and meropenem, and resistant to ampicillin. In light of antibiotic susceptibility, vancomycin was stopped, and therapy maintained with gentamicin and meropenem. On the same day, TFU showed an isolated temporal abscess, with follow-up TFU showing multiple bilateral abscesses more numerous in the right hemisphere (figure 1). On day 9 , abdominal ultrasound revealed one hepatic abscess.

On day 8, EEG showed a reduction in ictal events and a slight improvement in basal activity. Phenytoin was added to the drug regimen due to the presence of ictal events on EEG although no clinical convulsions were seen.

Ventilation, dopamine and morphine were stopped on day 12 and on day 15 clinical stability was achieved. A lumbar puncture was carried out, and cerebral spinal fluid (CSF) analysis revealed 61 cells (lymphocytes), proteins $201 \mathrm{mg} / \mathrm{dl}$ and glucose $26 \mathrm{mg} / \mathrm{dl}$. CSF culture was negative. Cefotaxime was added to the previous therapy and gentamicin was stopped.

TFU and cranioencephalic MR showed multiple cerebral abscesses on day 15 (figure 2). On day 16, neurosurgical evaluation ruled out the possibility of surgery due to the multiple lesions and the absence of mass effect.

Abdominal ultrasound on day 22 showed a reduction in the diameter of the hepatic lesion with resolution on day 37.

EEG on day 36 revealed well-structured electrogenesis with slight multifocal paroxysmal activity more persistent on the right temporal channels.

A 6-week course of intravenous antibiotic therapy was completed. TFU and cranioencephalic MR at the time of discharge showed multiloculated cystic encephalomalacia and diffuse hygroma (figure 3).

\section{OUTCOME AND FOLLOW-UP}

Currently, the patient is 14 months old and presents axial hypotonia and decreased number of movements but is able to feed herself. She has accomplished all neurodevelopmental milestones expected of her age.

Ophthalmological and auditory evaluation were normal. There were no further clinical convulsions and EEG was normal at 5 months of age. She is being weaned off anticonvulsant therapy.

The first twin and the mother were clinically asymptomatic during the entire period and analytical evaluation showed negative infection parameters and negative blood culture for the second twin.

\section{DISCUSSION}

$C$ koseri is a very rare cause of neonatal meningitis and infection is frequently associated with cerebral abscesses. Only a few cases have been reported. ${ }^{3-11}$

The reason why Citrobacter particularly affects neonates is not known, nor why it preferentially involves the central nervous system (CNS). ${ }^{3}$ A specific $32-\mathrm{kD}$ outer membrane protein may play a role as a neuro-virulent factor, providing Citrobacter tropism for the CNS and thus causing meningitis and cerebral abscesses. ${ }^{3}$

Around $76 \%$ of newborns with $C$ koseri meningitis develop brain abscesses $^{2}$ in contrast to other types of Gram-negative meningitis where this occurs in only $10 \%$ of cases. ${ }^{3}$ Globally, meningitis has a death rate of around $10 \%$, rising to $30 \%$ when Citrobacter is the agent. ${ }^{4}$

In this case, the newborn became symptomatic on day 3 and $C$ koseri was isolated from blood culture from the same day. The source of infection was not established. Other case reports describe symptomatic Citrobacter infection occurring from day 1 to day $60^{57}$ and both vertical and 


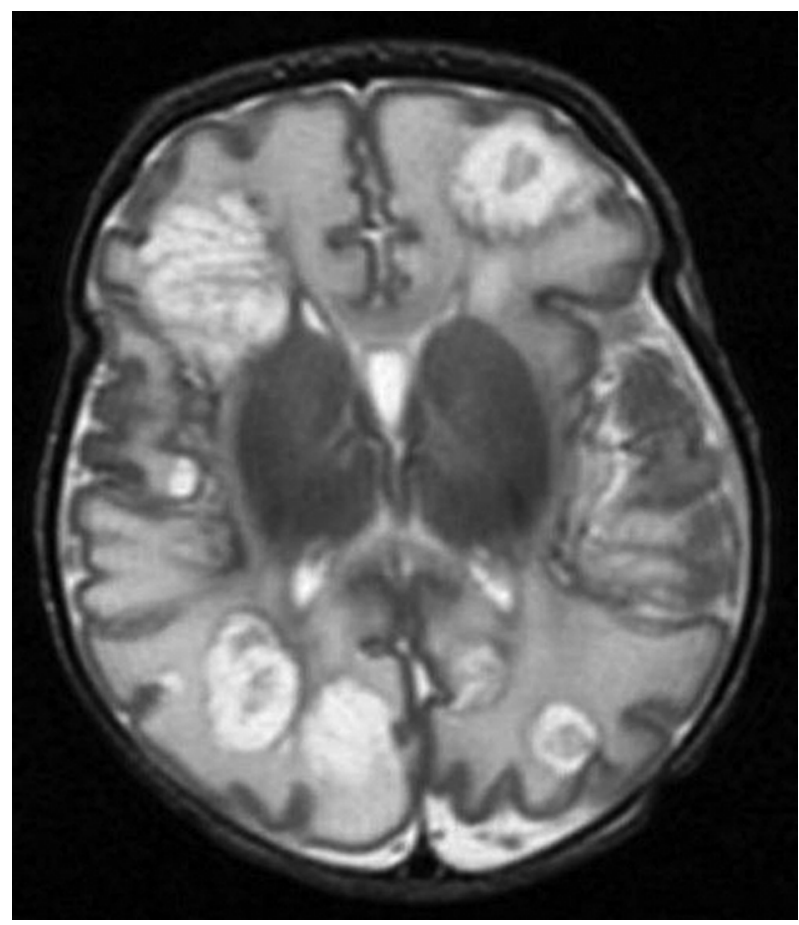

Figure 2 Cranioencephalic MR image showing multiple cerebral abscesses.

nosocomial transmission have been described. ${ }^{7}$ There is one report of Citrobacter infection in twins: twin one was symptomatic and twin two was asymptomatic, but both had Citrobacter isolated from CSF culture. ${ }^{2}$

Vertical transmission could not be established in the case described here. The mother was asymptomatic throughout pregnancy and after delivery, there were no signs of vaginal or intestinal infection or chorioamnionitis and the amniotic membranes were ruptured intrapartum. From the 26th week of gestation, the newborn showed growth restriction, which can occur in a twin pregnancy due to events other than infection. The second twin had normal growth during gestation and showed no adverse clinical events in the neonatal period. He was clinically asymptomatic, and blood analysis and culture were negative.

Nosocomial infection was also not proven. A venous umbilical catheter was present from day 1 to day 6 and no inflammation of the skin or umbilical cord were seen during this time. Culture from the catheter was negative. There were no other cases of Citrobacter infection among the neonates or the healthcare professionals, although a routine search (hands, surfaces and liquids) for this agent was not performed.

In a newborn diagnosed with Citrobacter infection, a lumbar puncture is mandatory due to the need for early diagnosis and appropriate treatment in case of cerebral abscesses. CSF culture remains the gold standard for the diagnosis of meningitis ${ }^{12}$; other CSF parameters should be interpreted with caution due to their high variability. ${ }^{12}$

In this case, lumbar puncture could only be carried out on day 12 due to haemodynamic instability; nevertheless, CSF cell count and chemical assays suggested meningitis. This reinforces the need for lumbar puncture in every newborn with sepsis, since the symptoms and signs

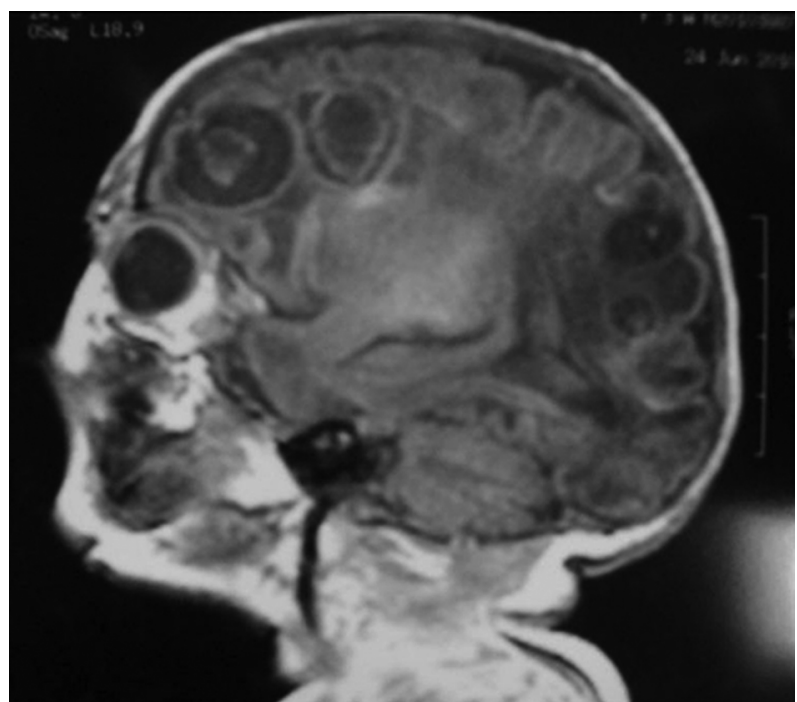

Figure 3 Cranioencephalic MR image showing multiloculated cystic encephalomalacia.

of meningitis in newborns are not specific. ${ }^{12}$ Indeed, CSF culture can be negative due to previous antibiotherapy, as in our case where the newborn had been exposed to 9 days of meropenem and gentamicin and a short course of vancomycin.

According to Gaschignard et al, neonatal meningitis has a death rate of $13 \%$ and up to $25 \%$ in preterm or small for gestational age infants, regardless of aetiology. ${ }^{13}$ Tatishvili et al described the outcome of bacterial meningitis according to the time of disease onset (before $72 \mathrm{~h}$ of life), maternal chronic infection and brain injury in newborns. ${ }^{14}$ These characteristics were predictors of severe complications in neonatal bacterial meningitis, such as brain abscess, ventriculitis, neonatal seizures, coma and neutropenia, and ultimately predictors of severe neurodevelopmental outcome. ${ }^{14}$ Gram-negative bacillary meningitis was also associated with worst prognosis ${ }^{15}$ as well as seizures for $>72 \mathrm{~h}$, presence of coma, use of inotropic agents and leucopenia. ${ }^{16}$

After meningitis is diagnosed, antibiotics should be selected according to CNS penetration and the spectrum of action. Traditionally, a combination of third generation cephalosporins and aminoglycosides is chosen, ${ }^{3}$ 5-7 although aminoglycoside resistance has been reported. ${ }^{17}$ In such cases, amikacin is superior to gentamicin. ${ }^{6}$

Ciprofloxacin and meropenem may be valuable options due to their CNS penetration. ${ }^{3}$ Cloranfenicol, cotrimoxazol and imipenem-cilastatina are also valid choices, ${ }^{8}$ although cloranfenicol and ciproflocaxin are not usually used in newborns.

The length of antibiotherapy should be extended in Citrobacter meningitis to at least 21 days $^{7}$ and $4-6$ weeks if abscesses are present. ${ }^{5}$

In the present case, after confirmation of cerebral abscesses on cranioencephalic MR (day 15), therapy was adjusted according to Citrobacter antibiotic susceptibility. The authors believe this could have been accomplished earlier as soon as the abscesses were seen on TFU (day 6), as earlier use of antibiotics with cerebral penetrance guarantees a better outcome. 


\section{BMJ Case Reports}

Urgent neurosurgical evaluation is required after a diagnosis of cerebral abscesses. Accessible cerebral abscesses and ventriculites with hydrocephalus should be drained. Surgical drainage should also be considered when there is no response to antibiotics. In the literature, the neurosurgical approach is associated with a better prognosis, especially when obstruction is present. ${ }^{5}$ Cerebritis and infarctation have no surgical indication. ${ }^{5}$ In this case, successful surgery was not feasible due to the high number of lesions and absence of mass effect.

Although no clinical convulsions were seen, phenytoin was added because of the abscesses and the persistent alterations on EEG despite maximum doses of phenobarbital.

In the case described, the neonate was a late preterm infant with growth restriction and altered diastolic flow in the umbilical artery. In light of the risk of necrotising enterocolitis (NEC), an umbilical catheter was placed and parenteral feeding started.

Our understanding of the pathogenesis of NEC remains incomplete, ${ }^{18}$ but it seems to be multifactorial and to involve an over-reaction of the immune system to an unspecific insult. ${ }^{19}$ The risk is inversely proportional to gestational age and birth weight, ${ }^{18}$ and fetal growth restriction and compromise may be additional specific risk factors. ${ }^{18}$ Careful feeding advancement may be an evidence-based strategy to decrease the incidence of NEC. ${ }^{19}$ However, delayed enteral feeding, the clinical importance of which is unclear, could harm functional adaptation of the gastrointestinal tract and prolong the need for parenteral nutrition with its attendant infectious and metabolic risks. ${ }^{20}$ The authors recognise the risk associated with umbilical venous catheter placement and that this procedure could have been detrimental to the newborn.

Currently, the newborn has axial hypotonia and a normal neurosensory evaluation, but neurological outcome is unpredictable and multi-disciplinary follow-up is necessary.

\section{Learning points}

- Citrobacter neonatal infection is rare and should be suspected if the clinical evolution is severe.

- The source of Citrobacter infection may not be evident, but vertical transmission and nosocomial infection have been reported.

- The use of an umbilical venous catheter is associated with risk of sepsis.

- Serial neuroimaging is mandatory in order to identify early cerebral complications.

- Third generation cephalosporins and aminoglycosides were used; surgical drainage should always be considered.

- Medical follow-up after discharge should be implemented to detect and treat sequelae.
Competing interests None.

Patient consent Obtained.

\section{REFERENCES}

1. Fenichel GM. Neonatal Neurology. Fourth Edition. Philadelphia, PA: Churchill Livingstone 2007

2. Etuwewe 0, Kulshrestha R, Sangra M, et al. Brain abscesses due to Citrobacter koseri in a pair of twins. Pediatr Infect Dis J 2009:28:1035.

3. Kariholu U, Rawal J, Namnyak S. Neonatal Citrobacter koseri meningitis and brain abscess. Internet J Pediatr Neonatol 2009:10:1.

4. McPherson C, Gal P, Ransom JL. Treatment of Citrobacter koseri infection with ciprofloxacin and cefotaxime in a preterm infant. Ann Pharmacother 2008;42:1134-8.

5. Martinez-Lage J, Azori L, Almagro M, et al. Citrobacter koseri meningitis: a neurosurgical condition? Eur J Pediatr Neurol 2009;I-4:2-4.

6. Samonis G, Karageorgopoulos DE, Kofteridis DP, et al. Citrobacter infections in a general hospital: characteristics and outcomes. Eur J Clin Microbiol Infect Dis 2009;28:61-8.

7. Doran TI. The role of Citrobacter in clinical disease of children: review. Clin Infect Dis 1999;28:384-94.

8. Azrak MA, D'Agustini M, Fernández Z, et al. [Citrobacter koseri brain abscess in an infant: case report and literature review]. Arch Argent Pediatr 2009;107:542-556.

9. Aller SC, Chusid MJ. Citrobacter koseri pneumonia and meningitis in an infant. J Infect 2002;45:65-8.

10. Alviedo JN, Sood BG, Aranda JV, et al. Diffuse pneumocephalus in neonatal Citrobacter meningitis. Pediatrics 2006;118:e1576-9.

11. Stallworth $\mathbf{L}$, Mani $C$, Murray D. Index of suspicion in the nursery Neoreviews 2005;6:e436-8.

12. Garages H, Moody A, Cotton M, et al. Neonatal meningitis: what is the correlation among cerebrospinal fluid cultures, blood cultures, and cerebrospinal fluid parameters? Pediatrics 2006;117:r 4.

13. Gaschignard J, Levy C, Romain 0, et al. Neonatal Bacterial Meningitis: 444 Cases in 7 Years. Pediatr Infect Dis J 2011;30:212-7.

14. Tatishvili NA, Sirbiladze TsV, Kipiani TB, et al. [Early predictors of neurodevelopmental outcome of neonatal bacterial meningitis]. Georgian Med News 2005;129:82-4.

15. May M, Daley AJ, Donath S, et al. Australasian Study Group for Neonatal Infections. Early onset neonatal meningitis in Australia and New Zealand, 1992-2002. Arch Dis Child Fetal Neonatal Ed 2005:90:F324-7.

16. Klinger G, Chin CN, Beyene J, et al. Predicting the outcome of neonatal bacterial meningitis. Pediatrics 2000;106:477-82.

17. Straussberg R, Harel L, Amir J. Long-Term outcome of neonatal Citrobacte koseri (diversus) meningitis treated with imipenem/meropenem and surgical drainage. Infection 2001;29:280-2.

18. Morgan JA, Young L, McGuire W. Pathogenesis and prevention of necrotizing enterocolitis. Curr Opin Infect Dis 2011;24:183-9.

19. Berman L, Moss RL. Necrotizing enterocolitis: an update. Semin Fetal Neonatal Med 2011;16:145-50.

20. Morgan J, Young L, McGuire W. Delayed introduction of progressive enteral feeds to prevent necrotising enterocolitis in very low birth weight infants. Cochrane Database Syst Rev 2011;3:CD001970. 
This pdf has been created automatically from the final edited text and images.

Copyright 2012 BMJ Publishing Group. All rights reserved. For permission to reuse any of this content visit http://group.bmj.com/group/rights-licensing/permissions.

BMJ Case Report Fellows may re-use this article for personal use and teaching without any further permission.

Please cite this article as follows (you will need to access the article online to obtain the date of publication).

Marecos CV, Ferreira M, Ferreira MM, Barroso MR. Sepsis, meningitis and cerebral abscesses caused by Citrobacter koseri. BMJ Case Reports 2012;10.1136/bcr.10.2011.4941, Published XXX

Become a Fellow of BMJ Case Reports today and you can:

- Submit as many cases as you like

- Enjoy fast sympathetic peer review and rapid publication of accepted articles

- Access all the published articles

- Re-use any of the published material for personal use and teaching without further permission

For information on Institutional Fellowships contact consortiasales@bmjgroup.com

Visit casereports.bmj.com for more articles like this and to become a Fellow 